\title{
Personalising and Crowdsourcing Stress Management in Urban Environments via s-Health
}

\author{
Achilleas Papageorgiou, Athanasios Zigomitros, Constantinos Patsakis \\ Department of Informatics, University of Piraeus, Greece. \\ apapageorgiou@ieee.org,\{azigomit,kpatsak\}@unipi.gr
}

\begin{abstract}
In an attempt to make our cities more sustainable and resource efficient, we are transforming them into what we now call Smart Cities. This transformation is heavily dependent on ICT, where a huge network of interconnected sensors and other devices is deployed to monitor many aspects of urban life in real-time. Recently, Solanas et al. [1] introduced the concept of Smart Health (s-Health) in which the resources of a smart city are exploited to facilitate the provision of healthcare services to citizens. In this position paper we explore the potentials of $s$ Health in stress management, providing a conceptual framework which could be used not only to monitor the stress level, but to provide more advanced ICT intervention.

Index Terms-Smart City, s-Health, stress management, mobile
\end{abstract}

\section{INTRODUCTION}

The wide adoption of mobile devices has triggered the development of a variety of new applications that have improved citizens' everyday lives. These changes are more evident in urban environments where there is a wealth of available resources. Currently, there are plenty of applications focusing on efficient time management exploiting geolocated information. Most of these applications are offered freely, and they monetize users' preferences, their demographic characteristics, as well as the extent of their user database by mainly providing them to advertising companies. Some applications may focus on specific groups of people, e.g. based on their health condition, and have positive impacts on their lives. These applications can be considered as successful business models exactly due to their targeted audience.

A big challenge of nowadays mobile applications providers, concerning big cities, is the satisfaction of their citizens' increasing everyday needs. Some citizens already use mobile applications in order to find the exact time that the bus will pass and buy their tickets ${ }^{1}$, the closest pharmacy to their location $^{2}$ or even the shortest route to their workplace ${ }^{3}$. All the above confirm that mobile applications are no longer something new for the average user, an assumption reinforced by their numerous downloads. Simultaneously wearable devices are becoming more and more accepted by modern users for their everyday life activities. Several applications are exploiting this new information to provide new features,

\footnotetext{
${ }^{1}$ https://play.google.com/store/apps/details?id=com.lothianbuses. lothianbuses

${ }^{2}$ https://itunes.apple.com/us/app/find-a-pharmacy/id358128302? $\mathrm{mt}=8$

${ }^{3}$ https://itunes.apple.com/us/app/inrix-xd-traffic-maps-routes/ id324384027?mt=8, http://www.google.com/mobile/maps/
}

more accurate measurements and even more personalised applications. The vast bulk of these applications is focused on sports and healthcare as most wearables can measure heartbeat rate, sweat and motion ${ }^{4}$.

All the above must be considered in the context of the Internet of Things (IoT), a large variety of devices connected to the Internet using mainly sensors. These devices in order to provide ubiquitous and context-aware services to humans, may interact and exchange information using several protocols, domains and applications [2], [3]. In most of the cases the use of the well-known (Machine to Machine) M2M protocols and (Wireless Sensors Networks) WSNs is needed in order to leverage low-power radios and multihop communication to cover large areas with small, inexpensive, autonomous sensor nodes and interconnection between machines with automation characteristics [3]. In the IoT the interconnected things can be vehicles, smart devices (smart phones, wearables), traffic lights or even buildings within a smart city.

In this position paper we attempt to bridge the latter with recent advances in Smart Cities technologies, and more precisely with the Smart Health concept [1]. Our scope is to exploit urban sensing with wearables to provide novel functionalities and more personalized user experience. More precisely, we introduce a novel scheme for context-aware mobile applications based on services with ubiquitous characteristics, as a s-Health solution, to citizens with stress or anxiety disorders. The idea is to extend the ICT intervention of such applications to provide users with solutions to their problems. In this regard, when citizens who suffer from the aforementioned disorders, are detected to face an issue, the platform will be present them solutions for mobility or interaction to decrease the risk of deteriorating their condition.

The rest of this work is organized as follows. Section II provides an overview of the current state of the art in mobile applications and wearables related to healthcare services. In Section III we illustrate two specific use-case scenarios and in Section IV we describe the conceptual approach of our framework. The article concludes with some remarks and ideas for future work.

\footnotetext{
${ }^{4}$ Microsoft-Band (https://www.microsoft.com/Microsoft-Band/en-us, Apple Watch (https://www.apple.com/watch/), Intel Basis Peak (https://www. mybasis.com/, Jawbone UP3(https://jawbone.com/store/buy/up3))
} 


\section{RELATED WORK}

Recent advances in portable devices, mainly smartphones [4], [5], [6] or wearable devices [7], [8], [9], have led companies to develop novel products related to health and wellbeing with more user-friendly and usable components such as smartwatches or fitness tracking devices. Today's smart devices base their services on software packages, namely applications. Currently there are plenty of applications which may help a user to monitor, manage or even diagnose stress levels. According to Muaremi et al. [10], these smartphone applications ${ }^{5}$ can be categorized into four main groups

1) Diaries refer to applications that can be used to collect and aggregate data related to stressful situations.

2) Guides are applications which offer several tricks and tips on how a user can handle stressful situations. Diaries and guides can be found to be combined in a unique application in order for an application to be more informative and useful for their users.

3) Relaxation applications provide sets of relaxation exercises which can help users to manage their stress levels.

4) Sensor measures are applications which offer a sensor based solution to users to track stress related behaviors.

There are several studies focusing on the of use smart devices to examine stress levels [11], [10], [12], or the relation between stress and daily habits or activities of users that can affect their mood [13], [14], [15]. Additionally, there are wearable devices which are becoming more widely known and in some cases accepted by users. Such devices can be considered the EEG headsets or the torso wearables. We refer to well known devices in the academic society, as for many years they have been used by researchers in order to monitor patients and produce valuable results. Their advanced monitoring abilities and interoperability with smartphones can be exploited in monitoring daily activities.

More precisely, many companies strive to embed sensors to their devices that can measure galvanic skin response. The idea is that sweat changes skin conductivity, so one could monitor these changes to determine how sweaty one is. Going a step further, there is a lot of research on the chemical analysis of body fluids, either invasive [16], [17] or non-invasive through e.g. patches or biosensing textiles [18], [19], [20], [21].

The EEG approach has also been used to measure stress levels, even if it was for children with Asperger syndrome [22]. While the experiments indicated that children with Asperger syndrome seem to have a greater reaction to stressful situation, it is apparent that EEG devices cannot currently be used in public as they might not be intrusive, nevertheless, they are not discreet enough. Therefore, users might become even more anxious, augmenting the negative impact that mobile devices can have such as stress, sleep disturbances or symptoms of depression [23], [24].

\footnotetext{
${ }^{5}$ https://play.google.com/store/books/details/Trina_Swerdlow_ Stress_Reduction_Journal?id=eTVhuMJXOdMC, https://play.google. com/store/apps/details?id=uk.co.oimedia.ibsdiary_android, $\quad$ https: //play.google.com/store/apps/details?id=com.pacificalabs.pacifica, https: //play.google.com/store/apps/details?id=com.Eliminate.Stress.management
}

\section{USE-CASE SCENARIOS}

According to [25], the urban way of life has high impact on mood and anxiety disorders compared with rural areas. To highlight such differences we provide two use-case scenarios of our framework to highlight its usage.

Bob is a very busy professional and his job requires a lot of meetings across the city he lives in. Recently, some events led him to become very anxious and have a panic crisis. In several occasions he had to stop walking just to calm down and retrieve his body control while being in the middle of a very crowded and noisy place. It turns out the cause of his bad health is the noisy urban environment [26].

On the other hand Alice is a teenager that lives in a not crowded place, but in order to go to her school or to participate to sports activities she has to move within very crowded and noisy streets that often make her feel anxious. This daily routine has a negative impact to her mental health and is very likely that she will face unwanted situations if she cannot avoid moving within stressful places.

In both cases one could argue that the solution would be to move away from the city. Nevertheless, personal, economic and social factors often prohibit such actions as the impact to other parts of the life could be detrimental. Moreover, such symptoms can be temporary, therefore such solutions are not the best. It would be greatly beneficial for both Alice and Bob to have an application that can detect when they are more fragile and provide an intervention with bigger impact.

\section{CONCEPTUAL APPROACH}

One of the basic problems in current state of the art is that the applications do not unlock the full potentials of the devices nor do they provide advanced intervention. More precisely, one could argue that the input is personal, that is the apps only process a single user's input, while the intervention is generic, it applies to everyone without personalisation, and is not predictive nor proactive.

Our conceptual approach, depicted in Figure 1 provides a more holistic approach than current state of the art. More precisely, the data is supplied by three sources:

1) the user, via data input and wearables

2) urban sensors, for instance pollution measurements, traffic and crowd sensors, and

3) crowd sourced data.

The idea is to use the personal data to determine the user's sentimental condition and the other data sources for prevention. For instance, the application can determine via the user's wearables that the user is feeling distressed by the change in galvanic skin response, without actual change in the temperature or movement of the user. Most applications currently are bound to this result and do not extend it further. Their intervention would be a simple poke to the user to perform some relaxation exercises. Therefore, this is were our conceptual framework starts to differentiate.

Firstly, the application would try to reroute the user, similarly to the concept of [27]. Instead of letting the user continue 


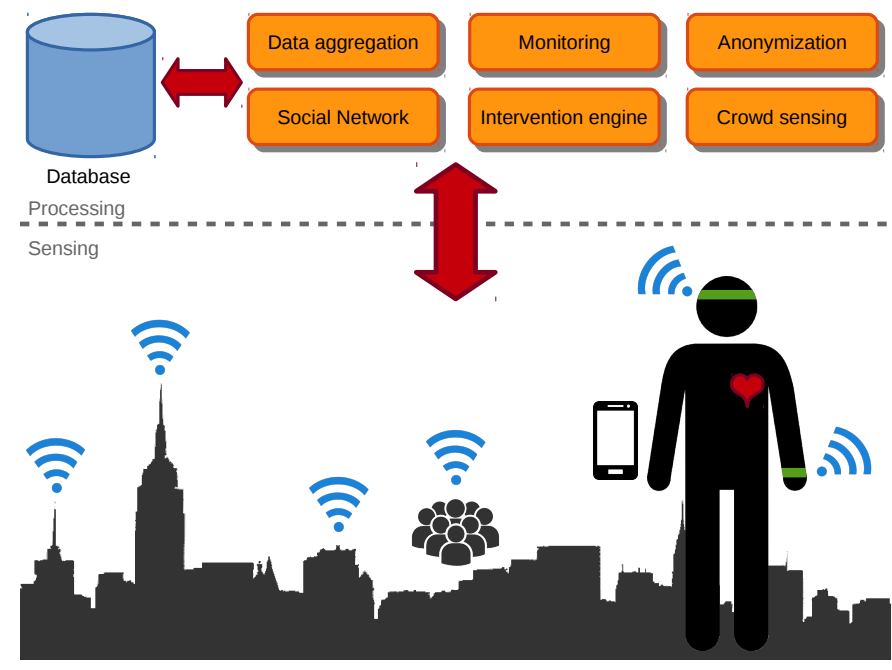

Fig. 1. A conceptual approach of the proposed scheme.

his course to his destination, the application would propose a different route to avoid locations that may increase her tension and lead to her collapse. To this cause, the application would monitor user's activity and routine to learn common routes. Based on the latter, the application would try to get measurements from urban sensors regarding noise levels, crowdedness etc and aggregate them. Based on these measurements, the application could determine alternate route to the user, which might be a bit more lengthy, but could be less stressful.

The social network engine could provide further assistance to the user. For instance, if other users share their location, the application could notify her of friends who are in proximity, events in the city or even daily news and hot stories from the media to relax the user. Depending on the user preferences the application could change the music and loudness to more soothing, as many users commute wearing ear-pads and listening to music.

Finally, the crowd sensing [28] aspect of the framework complements the sensing and prevention features. Users, could provide additional information to the platform to facilitate the others. For instance, they could share areas that made them feel distressed to notify others, provide audio input to facilitate noise mapping or even images to help in finding crowded or less crowded place. The crowd sensing approach has already been used in psychological trials in projects such as "Track your Tinnitus" $"$ providing valuable input for further research.

To provide all this functionality, the system is conceptualized to be divided into six main components as follows:

- The Monitoring engine is running on the user's device and keeps track of her location, movement, her routine, her vital measurements and preferences. Based on the readings, the platform will determine the user's stress level and decide whether an intervention is needed.

- The Data Aggregation engine is in charge of gathering urban data from smart city's sensors, aggregating them

\footnotetext{
${ }^{6}$ https://www.trackyourtinnitus.org
}

and proving an insight to the platform regarding probable issues and alternate routes.

- The Crowd Sensing engine provides additional input to the platform from other users. Users, acting as sensors may provide additional insight into events and locations that common sensors cannot. Therefore, they provide collective intelligence to the framework adding extra valuable content.

- The Intervention engine is providing the proactive mechanisms when the system detects that the user might face distress. The proactive mechanisms may range from music to social interaction, and from news and funny videos to games and relaxing exercises.

- The Social Network engine gathers information from social networks to provide the user some events, news and interaction with other users on the signal of the intervention engine.

- Finally, the Anonymization engine is in charge of the privacy. Its role is to hide users' identity in crowd sourced data as well as other sensitive information. For instance, hide the identities of individuals and their medical condition, hide faces from images or obfuscate users' location.

For interoperability, the platform should support many devices and protocols. Due to their processing power, smartphones can consume and produce information in many formats, nevertheless, sensors are very constrained in terms of computational resources. Therefore, the platform to be compliant with the IoT approach should cater for many protocols, specially lightweight ones to enable urban sensors to connect easily. Currently, there are plenty of middleware to facilitate this task, such as Mule ESB ${ }^{7}$.

\section{Challenges AND FUture WORK}

The unprecedented urbanisation is pushing towards the realisation of Smart Cities. The vast deployment of sensors can provide a real-time overview of the city and a wealth of information that researchers are trying to mine to extract new knowledge.

Nevertheless, urban life is quite complicated and more stressful than life in the suburbs. As a result, many people feel trapped in a rat race from which they cannot escape. In some cases, this feeling can lead to nervous breakdowns and stress crises. In this work we presented the concept of a novel framework that offers a more holistic approach to stress management compared to current state of the art, as it incorporates many technologies and could provide more advanced intervention.

The realization of the framework faces many challenges, such as data accuracy; sensors might not be properly calibrated or user contributed data might not be accurate, device interoperability, seamless user experience, user acceptance and privacy just to name a few. For device and protocol interoperability, platforms like Anypoint ${ }^{8}$ could facilitate the

\footnotetext{
${ }^{7}$ http://www.mulesoft.org/

${ }^{8}$ https://www.mulesoft.com/
} 
development while protocols such as Ardagna et al. [29] could provide users with the needed security and privacy. However, it has the potential to make significant impact in the lives of individuals and provide an insight in to how we should make our cities more sustainable and what are the key aspects in designing a human friendly urban environment.

\section{REFERENCES}

[1] A. Solanas, C. Patsakis, M. Conti, I. Vlachos, V. Ramos, F. Falcone, O. Postolache, P. A. Pérez-Martínez, R. Di Pietro, D. N. Perrea et al., "Smart health: a context-aware health paradigm within smart cities," IEEE Communications Magazine, vol. 52, no. 8, pp. 74-81, 2014.

[2] L. Atzori, A. Iera, and G. Morabito, "The internet of things: A survey," Computer networks, vol. 54, no. 15, pp. 2787-2805, 2010.

[3] J. Holler, V. Tsiatsis, C. Mulligan, S. Avesand, S. Karnouskos, and D. Boyle, From Machine-to-machine to the Internet of Things: Introduction to a New Age of Intelligence. Academic Press, 2014.

[4] E. Jovanov and A. Milenkovic, "Body area networks for ubiquitous healthcare applications: opportunities and challenges," Journal of medical systems, vol. 35, no. 5, pp. 1245-1254, 2011.

[5] P. C. CY, L. Qing, G. Hui, L. Wan-Hua, and Z. Yuan-Ting, "Wearable intelligent systems for e-health," Journal of Computing Science and Engineering, vol. 5, no. 3, pp. 246-256, 2011.

[6] M. M. Baig and H. Gholamhosseini, "Smart health monitoring systems: an overview of design and modeling," Journal of medical systems, vol. 37, no. 2, pp. 1-14, 2013.

[7] M. Chan, D. Estève, J.-Y. Fourniols, C. Escriba, and E. Campo, "Smart wearable systems: Current status and future challenges," Artificial intelligence in medicine, vol. 56, no. 3, pp. 137-156, 2012.

[8] J. R. Windmiller and J. Wang, "Wearable electrochemical sensors and biosensors: a review," Electroanalysis, vol. 25, no. 1, pp. 29-46, 2013.

[9] M. M. Baig, H. Gholamhosseini, and M. J. Connolly, "A comprehensive survey of wearable and wireless ecg monitoring systems for older adults," Medical \& biological engineering \& computing, vol. 51, no. 5, pp. $485-495,2013$.

[10] A. Muaremi, B. Arnrich, and G. Tröster, "Towards measuring stress with smartphones and wearable devices during workday and sleep," BioNanoScience, vol. 3, no. 2, pp. 172-183, 2013.

[11] G. Bauer and P. Lukowicz, "Can smartphones detect stress-related changes in the behaviour of individuals?" in Pervasive Computing and Communications Workshops (PERCOM Workshops), 2012 IEEE International Conference on. IEEE, 2012, pp. 423-426.

[12] A. Sano and R. W. Picard, "Stress recognition using wearable sensors and mobile phones," in Affective Computing and Intelligent Interaction (ACII), 2013 Humaine Association Conference on. IEEE, 2013, pp. 671-676.

[13] S. T. Moturu, I. Khayal, N. Aharony, W. Pan, and A. Pentland, "Sleep, mood and sociability in a healthy population," in Engineering in Medicine and Biology Society, EMBC, 2011 Annual International Conference of the IEEE. IEEE, 2011, pp. 5267-5270.

[14] S. T. Moturu, I. Khayal, N. Aharony, W. Pan, and A. S. Pentland, "Using social sensing to understand the links between sleep, mood, and sociability," in Privacy, Security, Risk and Trust (PASSAT) and 2011 IEEE Third Inernational Conference on Social Computing (SocialCom), 2011 IEEE Third International Conference on. IEEE, 2011, pp. 208214.

[15] G. Chittaranjan, J. Blom, and D. Gatica-Perez, "Who's who with bigfive: Analyzing and classifying personality traits with smartphones," in Wearable Computers (ISWC), 2011 15th Annual International Symposium on. IEEE, 2011, pp. 29-36.

[16] T. Guinovart, A. J. Bandodkar, J. R. Windmiller, F. J. Andrade, and J. Wang, "A potentiometric tattoo sensor for monitoring ammonium in sweat," Analyst, vol. 138, no. 22, pp. 7031-7038, 2013.

[17] A. J. Bandodkar, D. Molinnus, O. Mirza, T. Guinovart, J. R. Windmiller, G. Valdés-Ramírez, F. J. Andrade, M. J. Schöning, and J. Wang, "Epidermal tattoo potentiometric sodium sensors with wireless signal transduction for continuous non-invasive sweat monitoring," Biosensors and Bioelectronics, vol. 54, pp. 603-609, 2014.

[18] P. Salvo, F. Di Francesco, D. Costanzo, C. Ferrari, M. G. Trivella, and D. De Rossi, "A wearable sensor for measuring sweat rate," Sensors Journal, IEEE, vol. 10, no. 10, pp. 1557-1558, 2010.
[19] S. Coyle, K.-T. Lau, N. Moyna, D. O'Gorman, D. Diamond, F. Di Francesco, D. Costanzo, P. Salvo, M. G. Trivella, D. E. De Rossi et al., "Biotex-biosensing textiles for personalised healthcare management," Information Technology in Biomedicine, IEEE Transactions on, vol. 14, no. 2, pp. 364-370, 2010.

[20] M. Caldara, C. Colleoni, E. Guido, V. Re, and G. Rosace, "Development of a textile-optoelectronic ph meter based on hybrid xerogel doped with methyl red," Sensors and Actuators B: Chemical, vol. 171, pp. 10131021, 2012.

[21] A. J. Bandodkar and J. Wang, "Non-invasive wearable electrochemical sensors: a review," Trends in biotechnology, vol. 32, no. 7, pp. 363-371, 2014.

[22] S. Tiinanen, A. Matta, M. Silfverhuth, K. Suominen, E. JanssonVerkasalo, and T. Seppanen, "Hrv and eeg based indicators of stress in children with asperger syndrome in audio-visual stimulus test," in Engineering in Medicine and Biology Society, EMBC, 2011 Annual International Conference of the IEEE. IEEE, 2011, pp. 2021-2024.

[23] S. Thomée, A. Härenstam, and M. Hagberg, "Mobile phone use and stress, sleep disturbances, and symptoms of depression among young adults-a prospective cohort study," BMC public health, vol. 11, no. 1, p. 66,2011

[24] A. Johansson, S. Nordin, M. Heiden, and M. Sandström, "Symptoms, personality traits, and stress in people with mobile phone-related symptoms and electromagnetic hypersensitivity," Journal of psychosomatic research, vol. 68, no. 1, pp. 37-45, 2010.

[25] J. Peen, R. Schoevers, A. Beekman, and J. Dekker, "The current status of urban-rural differences in psychiatric disorders," Acta Psychiatrica Scandinavica, vol. 121, no. 2, pp. 84-93, 2010.

[26] S. A. Stansfeld and M. P. Matheson, "Noise pollution: non-auditory effects on health," British Medical Bulletin, vol. 68, no. 1, pp. 243-257, 2003.

[27] C. Patsakis, R. Venanzio, P. Bellavista, A. Solanas, and M. Bouroche, "Personalized medical services using smart cities' infrastructures," in 2014 IEEE International Symposium on Medical Measurements and Applications, MeMeA 2014, Lisboa, Portugal, June 11-12, 2014. IEEE, 2014, pp. 665-669. [Online]. Available: http://dx.doi.org/10. 1109/MeMeA.2014.6860145

[28] R. K. Ganti, F. Ye, and H. Lei, "Mobile crowdsensing: current state and future challenges," Communications Magazine, IEEE, vol. 49, no. 11, pp. 32-39, 2011.

[29] C. A. Ardagna, M. Conti, M. Leone, and J. Stefa, "An anonymous endto-end communication protocol for mobile cloud environments," IEEE Transactions on Services Computing, no. 3, pp. 373-386, 2014. 